\title{
reCHERches
}

Culture et histoire dans l'espace roman

Crise(s) dans le monde ibérique et ibéro-américain

\section{El metalenguaje gramatical verbal en el Arte de hablar bien francés de P.-N. Chantreau (1781) para uso de los españoles: ¿se puede hablar de crisis terminológica?}

Irene Valdés Melguizo

\section{(2) OpenEdition}

\section{Journals}

Edición electrónica

URL: https://journals.openedition.org/cher/4039

DOI: $10.4000 /$ cher.4039

ISSN: 2803-5992

\section{Editor}

Presses universitaires de Strasbourg

\section{Edición impresa}

Fecha de publicación: 1 diciembre 2015

Paginación: 271-281

ISBN: 978-2-86820-913-9

ISSN: 1968-035X

\section{REFERENCIA ELECTRÓNICA}

Irene Valdés Melguizo, «El metalenguaje gramatical verbal en el Arte de hablar bien francés de P.-N. Chantreau (1781) para uso de los españoles: ¿se puede hablar de crisis terminológica?», reCHERches [En línea], 15 / 2015, Publicado el 01 diciembre 2021, consultado el 19 diciembre 2021. URL: http://journals.openedition.org/cher/4039 ; DOI: https://doi.org/10.4000/cher.4039 


\title{
El metalenguaje gramatical verbal en el Arte de hablar bien francés de P.-N. Chantreau (1781) para uso de los españoles: ¿se puede hablar de crisis ${ }^{1}$ terminológica?
}

\author{
IRENE ValdÉs MeLGUIZO \\ Universidad de Granada - Université de Strasbourg
}

\begin{abstract}
A nalizamos en este artículo el metalenguaje gramatical verbal contenido en la gramática: el Arte de hablar bien francés (1781), de Pierre-Nicolas Chantreau, observando si hay innovación en relación a la terminología gramatical heredada del latín. Igualmente efectuamos algunas observaciones en gramáticas ${ }^{2}$ del francés editadas en Francia y en España en la época sin olvidar la Gramática de la Real Academia Española $(1771)^{3}$ para contextualizar y poder comprender los cambios producidos en el Arte de Chantreau.

El Arte de Chantreau es una gramática de referencia ineludible para comprender la evolución de la herramienta gramatical destinada a la enseñanza del francés a alumnos españoles a finales del siglo xviII y a lo largo del siglo XIX no solamente por el número de reediciones, reimpresiones y adaptaciones que ha conocido ${ }^{4}$ sino también por la constitución, según su modelo, de una manera de hacer (o método) llamado «tradicional» de la enseñanza del francés.
\end{abstract}

1 El término crisis lo comprendemos en nuestro estudio como la adaptación, transposición de los términos gramaticales en relación al verbo en la gramática de Chantreau. Se trata de adaptaciones, innovaciones que este gramático ha tenido que superar para poder adaptarse a los problemas de enseñanza/aprendizaje de una lengua extranjera considerando la lengua materna de su alumnado.

2 Consultar Referencias bibliográficas - Fuentes primarias.

3 Hemos efectuado una selección representativa de dichas obras porque un estudio completo de todas las obras de la época excedería los límites de dicho artículo.

4 Reediciones/reimpresiones de la gramática de P.-N. Chantreau: 1786, 1797, 1824, 1826, 1841, 1848, 1849, 1850, 1856, 1857, 1863, 1864, 1875, 1875, 1881, 1885, 1926, 1926, en las ciudades de Madrid, Barcelona, Reus, Lyon, Toulouse, Perpignan,

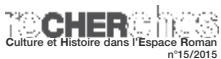


Se trata de una gramática redactada en español, es decir, en la lengua del alumnado. Este no es un hecho sin interés: ¿cómo hacer comprender, etapa necesaria y previa, términos y nociones desconocidas para el alumnado, así como el conjunto de la descripción gramatical, si no es gracias a su lengua materna?5

Nuestro análisis se limita a una cuestión precisa: nos centraremos únicamente en los términos gramaticales relativos al verbo por ser considerado este una parte esencial de la oración. Situaremos no obstante dicho estudio dentro de un enfoque comparativo: las relaciones entre la terminología utilizada para describir el verbo en francés y la terminología utilizada para describir el verbo en español, por cuanto los alumnos a quienes está dirigida la gramática francesa son alumnos españoles, que cuentan en principio con una formación gramatical previa, en cuanto a la lengua latina y española.

Dividimos nuestro estudio en los siguientes subcapítulos, que se corresponden con nuestro propio modo de proceder metodológico:

- Presentación del sistema verbal en su conjunto.

- Determinación de las nociones implicadas por los términos utilizados.

- Reflexiones acerca del papel asignado a la adquisición/aprendizaje del metalenguaje gramatical.

Este planteamiento se hará desde el punto de vista de la puesta en crisis de la terminología empleada.

\section{Sistema verbal y metalenguaje gramatical}

Como han destacado los especialistas en historiografía lingüística (Besse \& Porquier 1991; Caravolas 1994; Germain 1993; Kukenheim 1932; Stefanini 1994, entre otros), el metalenguaje gramatical constituye un conjunto terminológico relativamente estable (especialmente en cuanto a las grandes divisiones tales como las partes del discurso). Ahora bien, tras la gramatización de las lenguas vernáculas a partir del siglo XVI, surgen propuestas novedosas, puesto que las lenguas vernáculas no siempre se adaptan al marco terminológico del latín, o bien al desarrollarse partes nuevas de la descripción gramatical como la sintaxis (véase Chevalier [1968] 2006; Seguin 1993). De este modo, se constituyen unas tradiciones lingüísticas «nacionales» en las que tanto la terminología como la aprehensión de los conceptos gramaticales manifiestan ciertas peculiaridades. Nuestro estudio pretende, de modo concreto, poner de relieve algunas de ellas (en cuanto a la terminología verbal), utilizando como objeto de análisis un instrumento particular: una gramática para enseñar francés a hispanófonos, cuyo

Bordeaux, París, Bruselas... Las modificaciones que se producen entre unas y otras son muy importantes (véase la tesis de Moreu 1988) y el estudio de Fernández Fraile \& Suso López 1999).

5 Lancelot es el primer gramático al romper con la tradición y redactar una gramática latina en francés («mise en françois») (1644: Dédicace au roi). En 1660, publica una gramática de español también redactada en francés. 
autor tiene, forzosamente, que poner en paralelo las tradiciones gramaticales de ambas lenguas.

Una diferencia notable entre el Arte de Chantreau y las gramáticas anteriores editadas en España (Núñez 1728; Galmace [1745] 1780; Roca 1740) consiste en el afán definitorio constante con respecto a los términos gramaticales utilizados. Explica el porqué en el prólogo:

He formado una lista de los términos usados por los Gramáticos para la explicación de sus reglas; las definiciones de los unos van colocadas a la cabeza de sus respectivos capítulos; y quedan incluidas en dicha lista las de los demas: el motivo de esta división ha sido, apartar de la vista del lector instruido algunas definiciones prolixas, como las de los tiempos de los verbos \&c. y formar en la referida lista una especie de depósito donde pueda acudir el que las ignorase. (Chantreau 1781: XIV)

Chantreau procura de este modo adaptarse al variado " público » que era susceptible de utilizar su gramática: un público ya " instruido » o latinizado, conocedor del metalenguaje gramatical gracias al estudio del latín o de la lengua materna, y un público carente de formación gramatical. Y más allá de esta razón práctica, al señalar que es muy conveniente llevar «ya sabidos por su Gramática propia los principios comunes á todas las lenguas» (Chantreau 1781: III), no hace sino resaltar la necesidad de un enfoque contrastivo entre la lengua materna y la lengua que se aprende:

la única tarea de una Gramática, escrita para la enseñanza de una segunda lengua, debería ser el mero Analisis de las diferencias que se encontraren en el idioma materno, y el que se tratare de aprender. (Chantreau 1781: IV)

Tendremos que limitarnos a aportar los ejemplos más significativos, puesto que una presentación detallada de todas las diferencias existentes sería demasiado extensa.

\section{La clasificación de los verbos}

La tipología de verbos se basa en la clasificación tradicional, si bien introduce ciertos cambios. Lo significativo en este aspecto es que tal clasificación viene inducida por la definición propuesta del verbo, a la vez nocional y funcional. Así, según el tipo de relación establecida entre el sujeto y la acción, tenemos los verbos activos, pasivos, neutros: el verbo activo «indica una acción que pasa de un sugeto a otro: v.g. Dios castiga al pecador»; el verbo pasivo, "una acción recibida por el sugeto v.g. El pecador ha sido castigado por Dios»; y el neutro, «el ser o estar del sugeto v.g. Pedro es un perezoso, siempre está durmiendo» (1781: 92). Por otra parte, otros verbos expresan una acción reflexiva, o recíproca, cuando la acción recae en el sujeto; y finalmente, establecen el verbo impersonal (que se divide en propio e impropio), «quando no puede admitir por sugeto persona ó cosa determinada».

En cuanto a los cambios introducidos por Chantreau en este campo, destacamos desde el punto de vista terminológico la propuesta de la denominación 
de «verbes pronominaux» (pronominales) 6 para referirse a los verbos reflexivos o recíprocos:

b) Para que la reciprocación no sea ambigua, muchas veces es menester añadir al verbo otras palabras que la denoten, como: se mataron unos á otros, naturalmente entre sí etc. Véase la Gramática de la Real Academia Española, p. 59 y la del Abate Wailly, p. 95 [...].

c) Estos verbos dice la Gramática de la Real Academia Española, que nunca se usan sin pronombres, no deberían llamarse recíprocos ni reflexivos, sino pronominales. Véase dicha Gramática, pag. 59 y 60 (Chantreau 1781: 92, notas $b$ y $c$ ).

Así como la denominación de « intransitivos » para con los verbos neutros:

Muchos Gramáticos llaman los verbos neutros intransitivos, porque lo que expresa su significación no pasa a otra cosa: nacer, vivir, morir, etc. Véase la Gramática de la Real Academia Española, pag. 58. (Chantreau 1781: 92, nota a)

La definición de la GRAE es en efecto la siguiente:

No siendo pues estos verbos ni recíprocos ni reflexivos, debiera aplicárseles otra denominación y ninguna les convendría más que la de pronominales, como se ha dicho porque no pueden usarse sin pronombres [...]. No obstante estas razones ha prevalecido el uso de llamarlos recíprocos, y entendido así, no hay inconveniente en usar de esta denominación, pues por verbos recíprocos entenderemos lo mismo que por verbos pronominales. (GRAE 1771: 61)

Es de resaltar que Chantreau manifiesta en su texto una actitud prudente: no impone una terminología, sino que señala que la misma es utilizada («muchos gramáticos...») o recomendada («no deberían llamarse... sino...»). En ambos casos, el término propuesto es más transparente: en el primer caso, el criterio formal añadido (el verbo pronominal es aquel en que concurren dos pronombres) simplifica tanto el dispositivo clasificador como el reconocimiento de tal tipo de verbos, al eliminar la distinción entre reflexivo o recíproco; en el segundo, el término «intransitivo» entra a formar parte de un nuevo binomio (transitivointransitivo). Además de esta característica ilocutiva-discursiva del texto de Chantreau, y de las ventajas lógicas para la comprensión gramatical, destaca que en ambos casos se apoye en la GRAE para el uso de ambas denominaciones, mostrando así tanto la voluntad como la necesidad de un enfoque contrastivo en el uso de la terminología, para que los estudiosos de la lengua francesa vean facilitado su aprendizaje, puesto que algunos de ellos al menos deben poseer ya algunos conocimientos gramaticales obtenidos con el estudio de la lengua materna, como señala en el prólogo (Chantreau 1781: III-IV).

La fundamentación de su terminología y de las definiciones con que la acompaña en la GRAE es una característica constante del Arte de Chantreau: sin salir del campo del verbo, vuelve a tomar la definición del verbo defectivo como

6 Este término aparece a principios del siglo XviII, en la gramática del abad Dangeau (Mertens 1968: 67). 
«el que carece de algunos tiempos o personas» (Chantreau 1781: 131), haciendo una referencia explícita a la GRAE. El texto al que Chantreau alude es el siguiente:

Los verbos impersonales se llaman tambien defectivos por el defecto ó falta que tienen de personas; pero teniendo ya aquellos su denominacion suficiente, seria mas propio limitar la de defectivos á los verbos que no solo carecen de primeras, y segundas personas, sino de algunos tiempos, como: placer, yacer. (GRAE 1771: 168)

\section{El modo}

Debemos destacar en primer lugar, como indican Besse \& Porquier, que:

si les catégories de personnes et de temps ont été, historiquement, peu contestées, il n'en va pas de même pour celles de mode ou de groupe. Dès le $\mathrm{xvI}^{\mathrm{e}}$ siècle, Scaliger se moque des subdivisions modales que proposent ses prédécesseurs et Sanctius l'approuve de considérer que «le mode dans le verbe n'(est) pas une distinction nécessaire» (cité par J.-C. Chevalier 1968: 175). Beauzée et nombre de grammairiens du XviII ${ }^{\mathrm{e}}$ siècle considèrent qu'on ne peut faire de ce qu'on appelle l'impératif un mode à part entière. Le conditionnel n’a longtemps pas été admis, non plus, comme un mode [...]. (Besse \& Porquier 1991: 50)

Dicho esto, el Arte de Chantreau presenta de manera muy sucinta, en el cuerpo de su gramática, la cuestión de los modos, indicando únicamente que hay cuatro modos (infinitivo, indicativo, subjuntivo e imperativo), llamados así «porque en cada una de estas divisiones la acción expresada por el verbo se demuestra de un modo diferente» (Chantreau 1781: 94). Esta exposición es completada no obstante en la Lista Alfabética final (Chantreau 1781: 244-251):

Infinitivo (modo de) es el que expresa y representa la acción indicada por el verbo, sin explicar quien la hace, ni el tiempo en que se hace. El infinitivo comer, expresa la acción de comer, pero sin indicar quando, ni quien come [...].

Indicativo (el modo de) es el que indica, demuestra, y afirma sencillamente las cosas, v.g. yo soy, tú amas.

Imperativo (el modo de) es el que sirve para mandar, como: trae papel, ven acá. etc. $[\ldots]$.

Subjuntivo (el modo de) llamado así porque un verbo en subjuntivo necesita otro verbo antecedente expresado ó suplido, que perfeccione ó determine su sentido: v.g. justo es que yo ame á quien me ama. Si se quita el antecedente justo es, el subjuntivo que yo ame, no tiene ya sentido perfecto ni determinado (Chantreau 1781: 246-247 y 250).

Podemos comprobar así que la denominación «modo optativo» (existente en las gramáticas anteriores: véase Billet, Núñez, Galmace y Roca) desaparece de la gramática de Chantreau, a favor del término «modo subjuntivo», y siguen en este caso igualmente la terminología de la GRAE.

En cualquier caso, la cuestión de los modos debía ser consideraba como «espinosa» por Chantreau, puesto que en la Lista Alfabética de los términos no define al «modo», y remite al texto de la gramática. Por otra parte, Chantreau no distingue el condicional como modo, sino como tiempo del verbo incluido en el modo Indicativo, señalando en una nota a pie de página lo siguiente: 
La Gramática francesa incluye este tiempo en el modo de indicativo, y la castellana lo adapta á una de las tres terminaciones del pretérito imperfecto del subjuntivo. (Chantreau 1781: 244)

Para la GRAE el condicional no está representado en la tabla de conjugación porque forma parte del pretérito perfecto del subjuntivo (GRAE 1771: 66).

\section{Los tiempos}

El término «tiempo» plantea un problema crucial, a saber, la divergencia entre su acepción usual (existen únicamente tres tiempos: presente, pasado y futuro) y su acepción gramatical; existen varios tiempos pasados, varios tiempos futuros, tiempos que no indican presente, pasado ni futuro (el infinitivo, el participio), etc. No podemos entrar en examinar el planteamiento de los gramáticos de la época al respecto (pues excede los límites del presente artículo), y nos contentaremos con la descripción que propone Chantreau acerca de los diferentes tiempos verbales y de su formación. Nos detendremos más detenidamente en tres cuestiones que están relacionadas entre sí: el dispositivo terminológico; el mecanismo de formación de los tiempos, con la distinción entre tiempos primitivos y derivados, y la cuestión de los «tiempos del pasado».

En cuanto al dispositivo terminológico, podemos observar que Chantreau establece una distinción en cinco tiempos primitivos

- el presente de infinitivo,

- el participio,

- el gerundio presente,

- las tres personas de singular del presente de indicativo,

- el pretérito definido o simple (Chantreau 1781: 105-106).

Interesa señalar que el «passé simple» de las gramáticas francesas editadas en Francia corresponde en Chantreau al «pretérito definido ó simple» (je fus - yo fuí ó estuve), y que el "passé composé» corresponde al "pretérito indefinido o compuesto» (j’ai été - yo he sido o estado). En la GRAE encontramos el término «pretérito perfecto» (amé), que se subdivide en «pretérito perfecto próximo» y «remoto»:

Si se considera [la acción de la que se trata] absoluta y perfectamente pasada, se llama pretérito perfecto, como: fuí, escribí. Este pretérito perfecto se divide en próximo, y remoto. Próximo es el que denota mayor proxîmidad de aquella accion ó suceso que el verbo significa con respeto al tiempo en que se refiere; y remoto, el que la denota menor: v. g. he visto al Rey, es pretérito perfecto próximo, porque denota que desde que le ví hasta quando lo digo ha poco tiempo: ví al Rey es pretérito perfecto remoto, porque denota que desde que le ví hasta quando lo digo puede haber pasado mucho tiempo. (GRAE 1771: 64-65)

Chantreau utiliza así el término «pretérito definido ó simple» y no «pretérito perfecto próximo» (como en la GRAE) como correspondiente a "passé simple», y el término "pretérito indefinido ó compuesto», y no "pretérito perfecto remoto» (como en la GRAE) como correspondiente a "passé composé». Este uso es 
tanto más sorprendente cuanto en la tradición de gramáticas francesas editadas en España encontramos una elección terminológica diferente: «pretérito» (o pasado) y «pretérito indefinito» en Billet (1708: 64 y 114-115); "pretérito perfecto» y "pretérito perfecto compuesto» en Núñez ([1728] 1791: 98-100) y en Galmace ([1845] 1780: 120, 135); «perfecto definido» y «perfecto indefinido» en Roca y María (1750: 173).

En la Lista Alfabética de los términos usados en las Gramáticas [...] (Chantreau 1781: 244-251), Chantreau sintetiza esta cuestión del modo siguiente:

Pretéritos, se llaman así los tiempos de un verbo que indican una cosa pasada, y se dividen en pretérito definido ó simple, pretérito indefinido ó compuestos, pretérito anterior, pretérito imperfecto, y pretérito plusquam perfecto [...]

Pretérito anterior, llamado por la Gramática Española, pretérito perfecto remoto. Se expresa con el pretérito simple de los auxiliares, y el participio del verbo que se les agrega. Yo hube visto. Los Franceses le llaman anterior, porque la accion que expresa ha de ser anterior á otra: v.g. Quando yo hube visto al Rey, me retiré: la acción de ver al Rey es anterior á la de retirarme" (Chantreau 1781: 249).

En la parte dedicada a la sintaxis en el Arte de Chantreau (parte tercera de su gramática), existe un capítulo especial dedicado al «uso de los pretéritos» (Chantreau 1781: 228) y en el que Chantreau se hace eco de esta divergencia terminológica:

La regla dada en la Gramática de la Real Academia Española, página 64, sobre el uso de los pretéritos, también tiene lugar, y se observa en francés, esto es, que es preciso usar del pretérito simple o definido (llamado en español remoto) para indicar un tiempo enteramente pasado, v.g. Ayer recibí la noticia de la muerte de mi padre - je reçus hier la nouvelle de la mort de mon père; $y$ no: j’ai reçu hier, etc. [...] Pero quando se quiere indicar una acción pasada en un tiempo que no está enteramente pasado, es menester usar del pretérito compuesto o indefinido (en español próximo) [...] (Chantreau 1781: 228).

Chantreau añade igualmente un comentario para marcar el distinto uso de los pretéritos en español y en francés:

Adviertase que, quando no se indica determinadamente el tiempo, ó está algo remoto, se usa en francés del pretérito compuesto con preferencia del simple (Chantreau 1781: 228).

\section{Tiempos primitivos y tiempos derivados}

La gramática de Chantreau se limita a distinguir desde un criterio formal los diferentes tiempos: «Mirados los tiempos en quanto á su forma, se dividen en simples y compuestos» (Chantreau 1781: 94); y, más adelante: " Se dividen los tiempos simples en primitivos y derivados »(Chantreau 1781: 105): las denominaciones son transparentes: los tiempos simples se expresan con una palabra, los compuestos se expresan con más de una palabra; los tiempos derivados se forman a partir de los tiempos primitivos. Debemos señalar que Chantreau sigue con ello la tradición gramatical occidental, puesto que 
ambas distinciones (simples-compuestos; primitivos-derivados) remontan a los gramáticos griegos y latinos (Maux-Piovano 2000: 106). Ahora bien, estos gramáticos distinguían únicamente tres tiempos primitivos, mientras que, a lo largo del siglo XVIII, las gramáticas de francés (editadas en Francia) imponen «un regroupement sur cinq formes de base appelées temps primitifs (présent, passé simple, infinitif, participe présent et passé) à partir desquels il était posible de construire l'ensemble de la morphologie verbale» (véase Chervel 1977: 86; Besse \& Porquier 1991: 50). A efectos pedagógicos, dicha distinción supone un avance notable, puesto que es suficiente estudiar los primeros para saber toda la conjugación de los verbos.

Chantreau se extiende sobre el procedimiento de formación de los tiempos derivados a lo largo de 4 páginas, explicando claramente su objetivo:

Mi intento es que, enseñando como se forman los derivados de los primitivos, no haya sino saber bien estos, para formar después con facilidad y prontitud todos los tiempos que corresponden á la conjugación entera de un verbo; cuyo exemplo he dado en la de porter. A este efecto pondré aquí un índice de todos los primitivos de las quatro conjugaciones; el que debe estudiarse con la mayor atencion, siendo llave de la conjugacion (Chantreau 1781: 105).

Existe así en Chantreau, en esta cuestión, una mentalidad racionalizadora: pretende hacer entender al alumno el mecanismo de composición de los verbos (en este caso, los tiempos), de tal modo que el esfuerzo memorístico quede reducido en favor de una comprensión lógica.

\section{Papel asignado a la adquisición/aprendizaje del metalenguaje del sistema verbal}

Podemos afirmar que la adquisición del metalenguaje gramatical es inseparable para Chantreau de una correcta aprehensión nocional de los diferentes términos, y que ésta constituye un factor fundamental en el aprendizaje de una lengua. Ello es así puesto que tales términos/nociones, como habían suficientemente mostrado los gramáticos franceses a partir de la Grammaire générale de Port-Royal (1660), constituyen «los principios que son comunes á todas las lenguas» (Chantreau 1781: III). Si se acepta esta premisa, deben admitirse consecuentemente dos derivadas:

a) por un lado, que es sumamente ventajoso para el correcto aprendizaje de una lengua «hacer principiar los estudios» por la propia lengua materna;

b) en segundo lugar, que "para pasar al estudio de una segunda lengua, mucho convendría el estar antes enterado de los principios de la materna». Chantreau enuncia una tercera: «que para la enseñanza de una segunda lengua, debería ser el mero análisis de las diferencias que se encontraren en el idioma materno, y el que se tratare de aprender» (Chantreau 1781: IV).

Chantreau refiere en su apoyo dos citas: una que extrae del Prólogo de la GRAE («Los que hubieren de emprender carrera literaria, lo conseguirán con 
mayor facilidad, llevando ya sabidos por su Gramática propia los principios comunes á todas las lenguas»), y otra que extrae de la dedicatoria que Wailly dirige en su gramática a la Universidad de París, en la que se concreta la utilidad de tal modo de proceder:

Comme l'experience vous à appris que les elements, et la pluspart des regles du discours, sont communs à toutes les langues, c'est par les principes du François que vous commencez vos instructions. Létude par ce moyen ne rebutera pas les jeunes gens; parce que pour leur apprendre les regles de la langue maternelle il ne s'agit que de les rendre attentifs à des choses qu'ils savent deja confusement; et quand ils connoisent les principes de leur langues, il leur est aisé d'en faire l'aplication à celle de Athenes et de Rome. Vease Grammaire de M. l'Abbé de Wailly, pag. 4, edition 7 (Chantreau 1781: III-IV, nota $a$ ).

Chantreau extrae por su parte una tercera derivada (Chantreau 1781: IV), fundamentando así el enfoque contrastivo entre ambas gramáticas y ambas lenguas. Chantreau introduce así en su Arte frecuentes llamadas de atención hacia el funcionamiento de la lengua española en casos muy concretos (véase Fernández \& Suso 1999: 129-130).

De un modo más profundo, en nuestra opinión, la comprensión de la terminología gramatical, tal como se produce por medio de las definiciones de Chantreau, permite una conceptualización del funcionamiento de la lengua. Es decir, es el aprendizaje de la gramática la que fundamenta el resto de las actividades (o procedimientos de trabajo) que el maestro y el discípulo ponen en acción en la «clase». El «aprendizaje de la gramática» para Chantreau consiste en la comprensión de los términos gramaticales, de los mecanismos de composición de las palabras, de las regularidades morfológicas y de las similitudes entre ambas lenguas y sus diferencias, explicitadas gracias a los ejemplos correspondientes. Utilizamos el término «comprensión» en vez de memorización: la memorización es únicamente el resultado último, al que se llega tras los tres repasos recomendados por Chantreau. La discriminación del contenido en tres niveles, siendo únicamente el contenido del primer nivel el imprescindible, por tanto el único que debe ser memorizado, apoya la reducción del peso de la memorización a su justo término.

La gramática, para Chantreau, si es igualmente necesaria, lo es en sus elementos fundamentales, y no es concebida sino un medio para poder lanzarse a hablar y a escribir, simultáneamente al aprendizaje de la misma, puesto que las «reglas [...] se impresiona[n] mejor con la práctica, que con la teórica.» (Chantreau 1781: XX).

Hemos expuesto a lo largo de este estudio cómo el Arte de Chantreau constituye no solo un modelo en cuanto a la determinación del contenido gramatical (que el alumno debe aprender) sino a la terminología gramatical empleada.

Es por ello una figura clave en la configuración en España del llamado «método tradicional» de enseñanza del francés. Debemos decir a este respecto en primer lugar que la denominación «método tradicional» es ambigua: 
igualmente tradicional es el «método irregular». En segundo lugar, el «método tradicional» cubre una multitud de variantes, según países y períodos históricos, proponiéndose varias denominaciones para tales variantes históricas (véase Puren 1988: 44-61 y 62-75, y Fernández \& Suso 1999: 189-202, 348 y ss.).

Chantreau propone una terminología gramatical (nos hemos centrado únicamente en el verbo), así como unas definiciones de los propios términos prácticamente coincidentes; igualmente, hemos mostrado cómo dicha terminología, que se hereda de la transposición del metalenguaje del latín a las lenguas vernáculas, se ha ido adaptando al público hispanoparlante, a través de las diferentes gramáticas de francés editadas en España a lo largo del siglo XVIII. El papel de la GRAE en dicho proceso toma un carácter diferente con el Arte de Chantreau, al plantear este gramático la necesidad de un enfoque contrastivo entre gramáticas.

\section{Bibliografía}

\section{Fuentes primarias}

Billet, P.-P., [1673] 1708, Gramatica francesa, dividida en tres partes. La primera. Contiene los primeros rudimentos [...] La segunda. Comprehende vn tratado muy por extenso de la oracion, ò construccion, mas amplio que el de la primera ediccion, con vn paralelo de la eloquencia española, y francesa, y francesa, y española [...] La tercera. Contiene un arte poetica, o breve compendio de la poesia francesa [...], Madrid, Imprenta de Juan Garcia Infançon.

Chantreau, Pierre N., 1781, Arte de hablar bien francés, ó gramática completa [...], Madrid, Imprenta de Sancha.

Galmace, A., [1745]1780, Addiciones a la Gramática Francesa, que compuso el R.P. Nuñez, Madrid, Imprenta del Reino.

Núñez de Prado, J., [1728] 1791, Gramática de la lengua francesa, Madrid, Don Blas Román.

Real Academia Española, 1771, Gramática de la Real Academia española, Madrid, Joaquín de Ibarra.

Roca y María, S., 1750, Arte francés, o nuevo método facilísimo,para Leer, Hablar, y Escribir Francés, conforme a los más moderno [...], Barcelona, Francisco Surià.

\section{Fuentes secundarias}

Besse, H., \& Porquier, R., 1991, Grammaires et didactique des langues, SaintCloud, Hatier, Didier.

Caravolas, J.-A., 1994, Précis d'histoire de la didactique des langues I (1450-1700), Montréal, Les Presses Universitaires de Montréal.

Chervel, A., 1977, ...et il fallut apprendre à écrire à tous les petits français. Histoire de la grammaire scolaire, Paris, Payot. 
Chevalier, J.-C., 1968, Histoire de la syntaxe. Naissance de la notion de complément dans la grammaire française (1530-1750), Genève, Droz [Reedición Paris, H. Champion, 2006].

Fernández Fraile Ma E., \& Suso López, J., 1999, La enseñanza del francés en España (1757-1936), Granada, Método ediciones.

Germain, C., 1993, Évolution de l'enseignement des langues: 5000 ans d'histoire, Paris, Clé International.

Kukenheim, L., 1932, Contributions à l'histoire de la grammaire italienne, espagnole et française à l'époque de la Renaissance, Amsterdam, N. v. Nordhollandsche uitgevers-maatschappij.

Maux-Piovano, M.-H., 2000, Les débuts de la didactique de l'espagnol en France: les premières grammaires pratiques (1596-1660), Villeneuve d'Ascq, Presses Universitaires du Septentrion.

Moreu, N., 1988, Pierre-Nicolas Chantreau (1741-1808) et sa grammaire (Tesis doctoral), Barcelona, Universitat de Barcelona.

Mertens, J., 1968, Contribution à l'étude de la terminologie grammaticale française. La nomenclature $d u$ verbe chez les grammairiens français $d u X_{V I I}{ }^{e}$ siècle (Tesis doctoral), Leuven, Faculté de Philosophie et Lettres, KUL

Puren, C., 1988, Histoire des méthodologies d'enseignement des langues vivantes, Paris, Nathan / Clé international.

Stefanini, J., 1994, Histoire de la grammaire, Paris, CNRS Éditions. 